\title{
Targeted Therapy in Ovarian Cancer. A Comprehensive Systematic Review of Literature
}

\author{
YASSER DIAB $^{1}$ and MUSTAFA ZELAL MUALLEM ${ }^{2}$ \\ ${ }^{1}$ Department of Gynecology, Portland District Hospital, Victoria, Australia; \\ ${ }^{2}$ Department of Gynecology with Center for Oncological Surgery, \\ Campus Virchow-Klinikum, Charité Medical University, Berlin, Germany
}

\begin{abstract}
Background/Aim: We aimed to identify the most effectual groups of targeted therapies for ovarian cancer in recent clinical trials. Materials and Methods: A systematic literature review has been gathered on an inventive study design that comprises of five steps. This involves search for pertinent publications from 2010 to date in various accessible medical data bases, usage of inclusion and exclusion, appraisal of quality of the studies included, abstraction of the relevant data and intelligible amalgamation of the data abridged in an evocative and narrative style. Results: Three types of modalities of targeted-therapy drugs have been identified; angiogenesis inhibition, signal enzymes inhibition and apoptosis induction in the tumor cells. Conclusion: There has been a surge in clinical trials with drugs that specifically target signal enzymes, induce apoptosis and inhibit angiogenesis in site-specific ovarian cancer cells, which could be very promising to design a more efficacious protocol for treating the disease.
\end{abstract}

Targeted therapy is a recent drug innovation in the treatment of cancer. In contrast to chemotherapy, that involves employment of non-specific intracellular toxins that inhibit the process of cell division, targeted therapy, utilizes specific drugs that target exclusive molecular mechanisms that abet carcinogenesis, thereby reducing the side- effects due anti-

This article is freely accessible online.

Correspondence to: Dr. Mustafa Zelal Muallem, Charité University Medicine Berlin, Campus Virchow-Klinikum, Augustenburger Platz 1, 13353 Berlin, Germany. Tel: +49 30450664373, Fax: +49 30450564900,e-mail: Mustafa-Zelal.Muallem@charite.de

Key Words: Targeted therapy, ovarian cancer, angiogenesis inhibition, apoptosis induction, inhibition of signal enzyme mechanisms, review. mitotic damage to the normal cells, especially, in the bone marrow, digestive tract, and hair follicles.

The modality of these targeted-therapy drugs varies and includes signal enzymes inhibition, apoptosis induction and angiogenesis inhibition in the tumor. The arsenal of targeted therapy today comprises of drugs like imatinib mesylate, gefitinib, sunitinib and bortezomib.

Given the fact that ovarian cancer is one of the most deadly gynecological malignancies and the fifth leading cause of cancer-related mortality in women globally (1) there has been a recent surge in research efforts to explore new avenues and methods for treating ovarian cancer. Further, demonstration of the fact that a single short-term treatment of ovarian cancer cells with chemotherapy could result in a cancer stem cell (CSC)-like enriched residual population that would potentially generate significantly greater tumor burden has fuelled the search for better therapeutic manoeuvres for ovarian cancer (2).

There has also been a surge in clinical trials with drugs that specifically target signal enzymes, induce apoptosis and inhibit angiogenesis in site specific ovarian cancer cells, thus, utilizing the molecular profile of a patient's cancer to design a more efficacious protocol for treating the disease.

The present study is an inclusive review of topical literature on targeted therapy for the treatment of ovarian cancer.

\section{Search Strategy}

An inclusive literature search was accomplished by crossreferencing of keywords and ensuing second stage use of the references of the literature categorized in the preliminary sequence to condense the search operational. Consequent to the documentation of this principal collection of studies, formulation of the inclusion and exclusion criteria was achieved. 
The exploration included literature pertaining to targeted therapy in the treatment of ovarian cancer; the relevance of targeted therapy for the treatment of the disease; specificity of targeted therapy in ovarian cancer treatment; assessment of post-treatment response; enduring surveillance and recurrence of ovarian cancer. The evaluation terms included targeted therapy, targeted therapy in the evaluation of ovarian cancer, targeted therapeutics in ovarian cancer, recent innovations in targeted therapy for the treatment of ovarian cancer, evaluation studies on targeted therapy in ovarian cancer and comparison of assorted targeted therapeutics for ovarian cancer treatment.

One hundred and fifty three studies were obtained in the initial pursuit on recent trends and publications selected based on inclusion and exclusion criteria. One hundred and one studies were excluded as not pertaining to studies on ovarian cancer. Thirteen studies were excluded as not pertaining to specific manifestation, more recent diagnostic and treatment procedures. Seven studies were excluded as being editorials instead of being research publications. Two studies were excluded as letters to the editor. In the final selection, thirty studies were designated and implied as highly apposite to the current review.

\section{Methodology}

The systematic literature review has been erected on an inventive study design that comprises of five key steps. This involves search for pertinent publications in various accessible medical data bases, usage of inclusion and exclusion criteria for the selection of relevant publications, appraisal of quality of the studies included, abstraction of the relevant data and intelligible amalgamation of the data. The results of the studies in this review have been abridged in an evocative and narrative style. Quality evaluation has been done autonomously by two reviewers on quality of the methodology. The differences have been resolved by discussion with a third reviewer, wherever necessary in accordance with the guidelines of 'The Cochrane Reviewers' Handbook' guidelines (3).

Review guidelines relating to healthcare review including lucidity of the description of the problem, well defined objective stating the category of population, interventions and outcomes of interest and study designs have been incorporated. Since, it is an established fact that nonrandomized studies build estimates that imply more intense benefits of the effects of health care than randomized trials; substantial efforts to identify and include studies other than randomized trials have been placed in the study (3).

The search period has been from the year 2010 till date to guarantee more recent studies and technological trends. Search literature databases, websites and internet search engines employed included Biomed central, Cinahl, Cochrane
Library, Embase, Picarta, PubMed, SCI, www.doh.gov.uk, www.escriber.com, www.google.com and www.who.org.

Choice of inclusion criteria involved comparative study design, group protocol for treatment, assessment of posttreatment response and long-term surveillance in ovarian cancer, prevention and control, specific research studies on targeted therapy in ovarian cancer and targeted therapeutic awareness among the scientific population.

Selection exclusion criteria included editorials, letters, descriptions non-specific to targeted therapy and ovarian cancer treatment in the target population.

\section{Results}

Studies on anti-angiogenic therapeutics. Since angiogenesis plays a vital role in carcinogenesis, anti-angiogenic targeted therapy is principally based on the fact that therapeutics that inhibit the process of angiogenesis would retard the growth and progression of tumors (4). Apart from the monoclonal Vascular endothelial growth factor (VEGF) antibody Bevacizumab, numerous anti-angiogenic therapeutics for ovarian cancer patients have been evaluated in clinical trials. This evaluation has been, perhaps, due to the failure to demonstrate the efficacy of bevacizumab with reference to overall survival (OS) instead of just a demonstrated progression-free survival (PFS) in the target groups, in various studies including the International collaboration on ovarian neoplasms trial 7 (ICON7) and the complementary Gynecologic oncology group study 218 (GOG218) (for the first-line treatment) (5), the Ovarian cancer study comparing efficacy and safety of chemotherapy and antiAngiogenic therapy in platinum-sensitive recurrent disease (OCEANS) (for platinum-sensitive recurrence) (6), and the Avastin use in platinum-resistant epithelial ovarian cancer (AURELIA) trials (for platinum-resistant recurrence) (7).

These include those targeting extracellular receptors, those that interfere with VEGF receptors and multiple additional intracellular pathways that are VEGF independent.

A recent international, double-blind phase III antiangiopoietin therapy with trebananib for recurrent ovarian cancer (TRINOVA -1) trial in a cohort of 919 patients with recurrent ovarian cancer with a platinum-free interval $<12$ months and $\leq 3$ prior therapies has studied the inhibition of angiogenesis with a peptibody trebananib (AMG386) through the angiopoietin pathway by blocking the binding of the angiopoietins Ang1/Ang2 to the Tie2 receptor, thus, addressing a VEGF-independent, parallel anti-angiogenic pathway. This study has shown a significantly improved median PFS of 2.8 months [7.2 vs. 5.4 months; hazard ratio (HR) 0.66 ; $95 \%$ CI $0.57-0.77$; <0.001] with lesser VEGFassociated side effects such as hypertension, proteinuria, thromboembolic occurrences $(8,9)$.

Studies pertaining to the inhibition of angiogenesis by targeting multiple VEGF proteins in the VEGF pathway 
apart from VEGF gained importance recently with studies on the fusion protein aflibercept containing VEGF binding domains from extracellular regions of the VEGF receptor 1 (VEGFR-1) and VEGFR-2. The protein has been shown to have strong broad affinity towards VEGF-A, VEGF-B and have demonstrated a better control of malignant ascites with a reduction of the interval between paracenteses (e.g., 55.1 vs. 23.3 days, respectively; $95 \% \mathrm{CI}=10.6-53.1 ; p=0.0019)$ in phase II clinical trials in a cohort of relapsed ovarian cancer patients (10). Phase III clinical trials have not been in place perhaps due to reporting of Grade 3/4 adverse effects including hypertension in up to $27.5 \%$ of the cases, dyspnea in up to $20 \%$, proteinuria in up to $9.4 \%$ of patients accompanied with a higher rate of intestinal perforation (10).

Inhibition of angiogenesis by multikinase inhibitors has on the other hand shown promising results by intracellular blockade of signal transduction pathways instead of the pursuing the extracellular receptors discussed previously. An oral tyrosine kinase inhibitor targeting three different protein kinases (VEGFR, platelet-derived growth factor receptors (PDGFR), and C-Tyrosinkinase (c-KIT)) Pazopanib has been proven effective and currently is being tested in numerous phase II trials (11-14).

Nintedanib (BIBF 1120) targeting VEGFR, PDGFR, and Fibroblast growth factor receptors (FGFR) is yet another oral triple angiokinase inhibitor that is currently being studied as the first-line therapy of ovarian cancer. A randomized phase III trial in a cohort of 1,366 patients has shown that the primary endpoint (median PFS) was prolonged from 16.6 to 17.3 months $(\mathrm{HR}=0.84 ; 95 \% \mathrm{CI}=0.72-0.98 ; p=0.024)(15)$.

The oral tyrosine kinase inhibitor cediranib has been shown to be a potent inhibitor of all three VEGF receptors (VEGFR-1, -2, -3) and c-kit with pronounced selectivity for VEGFR-2 in an open-label phase II trial followed by a randomized, double-blind, placebo-controlled phase III trial. The results of a randomized phase II trial investigating the combination of cediranib $30 \mathrm{mg}$ daily and the Poly(ADPribose) polymerase (PARP) inhibitor olaparib $200 \mathrm{mg}$ twice dialy vs. olaparib $400 \mathrm{mg}$ twice dialy alone in 90 women with recurrent platinum-sensitive ovarian cancer and a deleterious germline Breast cancer gene $(B R C A) 1$ or 2 mutation has focused the attention on the drug further. The international randomized phase III Platine, avastin and olaparib in 1st line (PAOLA-1) trial initiated recently by the french Groupe d'investigateurs nationaux pour l'étude des cancers ovariens (GINECO) to investigate the combination of chemotherapy, anti-angiogenic therapy and PARP inhibitors in the first-line setting for ovarian cancer patients is worth the mention in this context (16-18).

Sunitinib, a multikinase inhibitor targeting VEGF receptors, PDGF receptors, stem cell factor receptor (KIT) and FMS-like tyrosine kinase-3 (Flt-3), is currently in phase II studies for ovarian cancer and recurrent disease (19).
Sorafenib is another oral multitargeted tyrosine kinase inhibitor blocking VEGFR2, VEGFR3, as well as PDGFR beta, Flt-3, and c-kit (26) that also has partial inhibitory effects on portions of the RAS/RAF/MEK/ERK signaling pathway that has been established to have a pivotal role in the development of ovarian cancer (20).

\section{Studies on Targeting Signaling Pathways}

Numerous studies have shown that targeting specific signaling pathways involved in cancer progression could be a promising molecular therapeutic choice in combating cancer in terms of inhibition of cell migration, invasion and tumor growth.

Recent studies have revealed that targeting GRB7/ERK/ FOXM1 signaling pathway impairs aggressiveness of ovarian cancer cells. Subsequent to the findings that inhibition of ERK signaling by U0126 (1,4-diamino-2,3-dicyano-1,4-bis (2aminophenylthio)butadiene) or PD98059 (2-(2-Amino-3methoxyphenyl)-4H-1-benzopyran-4-one) could reduce the level of FOXM1 in GRB7-overexpressing ovarian cancer cells, it is suggested that GRB7, ERK and FOXM1 are regulated in a cascade or orderly sequence. In vitro and in vivo studies have shown that inhibition of ERK activity by U0126 or PD98059, or decreased FOXM1 expression by Thiostrepton drastically inhibits cell migration, invasion and tumor growth (21).

Sufficient attention has been drawn on the Sphingosine kinase 1 (SPHK1), the enzyme that produces Sphingosine 1 phosphate (S1P). Studies have confirmed the extensive expression of SPHK1 in the tumor stroma of High-grade serous ovarian cancer (HGSC) and its pivotal role in the differentiation and tumor promoting function of cancerassociated fibroblasts (CAFs). These studies have further exhibited that pharmacological inhibition of SPHK1 in ovarian fibroblasts could attenuate TGF- $\beta$-induced expression of CAF markers, reducing their capacity to stimulate ovarian cancer cell migration and invasion. Elucidation of the mechanism by which SPHK1 mediates TGF- $\beta$ signaling through transactivation of S1P receptors (S1PR2 and S1PR3), leading to $\mathrm{p} 38$ MAPK phosphorylation and in vivo confirmation of reduction of tumor growth and metastasis in SPHK1 knockout mice have further widened the scope for further investigations on the potential of SPHK1 inhibition as a novel stroma-targeted therapy in HGSC cases (22).

It is imperative to mention the focus on targeting cyclindependent kinase pathways in the treatment of ovarian and other gynaecological cancers. The new class of targetedtherapeutic agents known as the CDK 4/6 inhibitors target the CDK $4 / 6$ kinases that promote transition through the cell cycle. Some of the oral $C D K 4 / 6$ inhibitors now under active clinical trials include palbociclib, abemaciclib and ribociclib and have been reported to have negligible toxicity (23).

A recent study has shown that a chemically synthesized isoflavone analog (RY-2f), suppressed PI3K/AKT/mTOR 
signaling and is a potential targeted therapeutic. In vivo studies have revealed that $R Y$ - $2 f$ could effectively block an A2780-induced xenograft tumor growth with no detectable toxicity in the animals at the therapeutic doses. Studies have also implied that that this isoflavone analog, inhibits ovarian cancer cell proliferation, blocks cell cycle in $\mathrm{G}_{2} / \mathrm{M}$ phase and induces cellular apoptosis through up-regulation of $\mathrm{p} 21$, cyclin B1, Bax, Bad and cleaved-PARP, and suppression of cyclin A, CDK2 and Bcl-2. An improved synergistic therapeutic effect of RY-2f and cisplatin has also been elucidated in cell proliferation and colony formation assays as well (24).

Importunate activation of STAT3 activated by janus family kinases (JAK) via cytokine receptors, growth factor receptor, and non-growth factor receptor tyrosine kinases is often detected in ovarian cancer. Moreover, studies have shown that activation of STAT3 mediates tumor cell proliferation, survival, motility, invasion, and angiogenesis, suppresses antitumor immune responses and supports tumorpromoting inflammation. An elucidation and evaluation study on targeted blockade of JAK/STAT3 signaling in inhibition of ovarian cancer is worth the mention in this context. Evaluation of AZD1480, a novel ATP-competitive JAK inhibitor, on cell viability, apoptosis, proliferation, migration, and adhesion of ovarian carcinoma cells in vitro and in vivo assessment of tumor growth, progression, gene expression, tumor-associated matrix metalloproteinase (MMP) activity, and immune cell populations in a transgenic mouse model of ovarian carcinoma has shown that AZD1480 treatment inhibits STAT3 phosphorylation, DNA binding, migration and adhesion of cultured ovarian carcinoma cells with a diminished ovarian tumor growth rate, volume, and ascites production. Thus, therapeutic pharmacologic inhibition of the JAK2/STAT3 pathway can disrupt ovarian tumor growth and warrants further investigation as a targeted therapy (25).

Studies involving the treatment of isolated tumor cells from the ascites of ovarian cancer patients and HEY ovarian cancer cell line with paclitaxel in a mouse xenograft model and in vitro assays have shown that the inhibition of the JAK2/STAT3 pathway in ovarian cancer results in the loss of cancer stem cell-like characteristics (2) and a reduced tumor burden. Subsequent to the findings that treatment of isolated tumor cells from the ascites of ovarian cancer patients and HEY ovarian cancer cell line with paclitaxel results in a CSC-like residual population which goes in tandem with the activation of Janus activated kinase 2 (JAK2) and Signal transducer/activation of transcription 3 (STAT3) pathway in paclitaxel surviving cells, it has been established that a low dose JAK2-specific small molecule inhibitor (Momelotinib) CYT387 $(1 \mu \mathrm{M})$ inhibits paclitaxelinduced JAK2/STAT3 activation and CSC-like characteristics in vitro and a reduced tumor burden in vivo (2).
Sufficient attention has been drawn on the phosphatidylinositol 3 kinase (PI3K) pathway, which is frequently altered in ovarian cancer patients. Quite a number of novel therapeutics including dual mTORC1/mTORC2, Akt, and PI3K inhibitors are under active investigation. Given the fact that a random inhibition of the $\mathrm{PI} 3 \mathrm{~K} / \mathrm{Akt} / \mathrm{mTOR}$ pathway in a nonspecific ovarian cancer cohort could have little or no effect, it is recommended to select specific histological types, like clear cell or endometrioid ovarian cancer with phosphatidylinositol-4,5biphosphate 3-kinase, catalytic subunit alpha (PIK3CA) and/or phosphatase and tensin homolog (PTEN) alterations in such studies. It is further suggested that the PI3K pathway inhibition could be more effective in combination with either chemotherapy or other targeted therapies, including MEK inhibitors, anti-angiogenic therapy in specific histological types of the ovarian cancer due to the intricacy of the PI3K signaling mechanism (26).

\section{Studies on Induction of Apoptosis}

Previous studies on apoptosis induction in cancer cells have demonstrated that an adenovirus type 5 E1A enhances the sensitivity of paclitaxel in paclitaxel-resistant HER-2/neuoverexpressing human ovarian cancer cells in vitro by inducing apoptosis. The studies have further elaborated that apoptosis induction is largely dependent on activation of the caspase-3 pathway (27).

A study designed to explore the effects of liposomesurvivin antisense oligonucleotide (Lip-ASODN) on the growth and apoptosis of human ovarian cancer cell lines, A2780 and SKOV3 and investigate the use of survivin as a therapeutic target on ovarian cancer has established that the overexpression of survivin leads to infinite carcinoproliferation, and survivin expression in the survivin-positive ovarian cancer cell line A2780 and SKOV3 cells could be significantly and gradually reduced when transfected with Lip-ASODN at concentrations of 200, 400 and $600 \mathrm{nM}$. The study has further shown that Lip-ASODN transfection induces greater apoptosis rates in the human ovarian cancer cell lines A2780 and SKOV3 $(p<0.05)(28)$.

Enzastaurin (LY317615.HCl), a selective inhibitor of protein kinase $\mathrm{C}$ beta (PKCbeta), is yet another entity that causes induction of apoptosis. Studies have shown that a combination of enzastaurin and pemetrexed inhibits cell growth and induces apoptosis of chemoresistant ovarian cancer cells regulating extracellular signal-regulated kinase $1 / 2$ phosphorylation (29).

Recent studies to evaluate the ability of metformin to induce apoptosis in epithelial ovarian cancer cell lines and to identify the pathways involved have established that metformin induced apoptosis in OVCAR-3 and OVCAR-4 cell lines in an Adenosine monophosphate activated protein 
kinase (AMPK)-independent mode and metformin would induce apoptosis in OVCAR-3 and OVCAR-4 cells by activating caspases 3/7, down-regulating Bcl-2 and Bcl-xL expression, and up-regulating Bax and Bad expression (30).

More recent studies have established that the targeted inhibition of FAK, PYK2 and BCL-XL synergistically enhances apoptosis in ovarian clear cell carcinoma cell lines. Tumor necrosis factor-related apoptosis-inducing ligand (TRAIL) is another targeted therapeutic strategy for ovarian cancer. A Mitochondrial division inhibitor-1 (Mdivi-1) has been shown to enhance the sensitivity of human ovarian cancer cells to death receptor ligands including TRAIL, FAS ligands, and TNF- $\alpha$. The absence of significant cytotoxic effects on non-transformed human cells with the combination of TRAIL and mdivi-1 enhances the scope further (31).

\section{Discussion}

Validation of the verity that short-term treatment of ovarian cancer cells with chemotherapy results in an enriched residual cancer stem cell (CSC)-like population that creates a potentially significant tumor burden and the anti-mitotic side- effects to the normal cells during chemotherapy has prompted the exploration of improved therapeutic stratagems for the treatment of ovarian cancer.

The synthesis and analysis of these results indicates that all the three modalities of the targeted therapy discussed viz., signal enzymes inhibition, apoptosis induction and angiogenesis inhibition in the cancer cells are quite effective with most of the therapeutics in phase II trials.

Inhibition of paclitaxel-induced JAK2/STAT3 activation with momelotinib - CYT387 and targeted blockade of JAK/STAT3 signaling with a novel ATP-competitive JAK inhibitor AZD1480 are noteworthy investigations. In vitro analysis of tumor xenografts at protein and mRNA levels have demonstrated a loss of CSC-like markers and CA125 expression in paclitaxel and CYT387-treated cell-derived xenografts, compared to paclitaxel only-treated cell-derived xenografts. Studies on the effects of AZD1480 on in vivo tumor growth and progression, gene expression, tumor-associated matrix metalloproteinase (MMP) activity, and immune cell populations in a transgenic mouse model of ovarian carcinoma have also demonstrated an inhibition of STAT3 phosphorylation, migration and adhesion of ovarian carcinoma cells, ovarian tumor growth rate, volume, and ascites production.

On studies targeting the induction of apoptosis, apoptosis induction by Mdivi-1 that enhances the sensitivity of human ovarian cancer cells to death receptor ligands including TRAIL, FAS ligands, and TNF- $\alpha$ is a recent significant investigation with an absence of sizeable cytotoxic effects on non-transformed human cells.

Studies on anti-angiogenic targeted therapeutics have brought forth numerous research and clinical trials targeting extracellular receptors, those that interfere with VEGF receptors and multiple additional intracellular pathways that are VEGF-independent. A double-blind phase III TRINOVA1 trial with a peptibody trebananib (AMG386) that acts through the angiopoietin pathway blocking the binding of the angiopoietins Ang1/Ang2 to the Tie2 receptor addressing a VEGF independent, parallel anti-angiogenic pathway; studies on the fusion protein aflibercept containing VEGF binding domains from extracellular regions of the VEGF receptor 1 (VEGFR-1) and VEGFR-2 in phase II clinical trial; inhibition of angiogenesis by multikinase inhibitors in multiple phase II clinical trials; an oral triple angiokinase inhibitor nintedanib (BIBF 1120) targeting VEGFR, PDGFR, and FGFR in phase III trial; The international randomized phase III PAOLA-1 trial initiated recently by the French Groupe d'Investigateurs Nationaux pour l'Étude des Cancers Ovariens (GINECO) to investigate the combination of chemotherapy, anti-angiogenic therapy and PARP inhibitors in the first-line setting for ovarian cancer patients are noteworthy investigations.

\section{Conclusion}

Corroboration of the fact that a single short-term treatment of ovarian cancer cells with chemotherapy could result in a cancer stem cell (CSC)-like enriched residual population that would potentially generate significantly greater tumor burden and failure to demonstrate the efficacy of key targeted angiogenesis inhibitors like bevacizumab in the overall survival (OS) instead of just a progression-free survival (PFS) in various studies including the ICON7 and the GOG218, the OCEANS and the AURELIA trials has fuelled the search for better targeted-therapeutic manoeuvres for ovarian cancer, resulting in an arsenal of novel successful targeted therapeutics that specifically target signal enzymes, induce apoptosis and inhibit angiogenesis in site-specific ovarian cancer cells more effectually.

\section{Conflicts of Interest}

No financial or personal conflict of interest by any of the Authors to declare.

\section{References}

1 Ferlay J, Shin HR, Bray F, Forman D, Mathers C and Parkin DM: Estimates of worldwide burden of cancer in 2008: GLOBOCAN 2008. Int J Cancer 127(12): 2893-2917, 2010.

2 Abubaker K, Luwor RB, Zhu H, McNally O, Quinn MA, Burns CJ, Thompson EW, Findlay JK and Ahmed N: Inhibition of the JAK2/STAT3 pathway in ovarian cancer results in the loss of cancer stem cell-like characteristics and a reduced tumor burden. BMC Cancer 14: 317, 2014.

3 Clarke M and Oxman A. Cochrane reviewers' handbook 4.2.0. 2003. Available online at: http://www.cochrane.dk/cochrane/ handbook/handbook.html. Accessed on Dec 5th 2016. 
4 Gavalas NG, Liontos M, Trachana SP, Bagratuni T, Arapinis C, Liacos C, Dimopoulos MA and Bamias A: Angiogenesis-related pathways in the pathogenesis of ovarian cancer. Int J Mol Sci 14(8): 15885-15909, 2013.

5 Burger RA, Brady MF, Bookman MA, Fleming GF, Monk BJ, Huang H, Mannel RS, Homesley HD, Fowler J, Greer BE, Boente M, Birrer MJ, Liang SX and Gynecologic Oncology Group: Incorporation of bevacizumab in the primary treatment of ovarian cancer. N Engl J Med 365(26): 2473-2483, 2011.

6 Aghajanian C, Blank SV, Goff BA, Judson PL, Teneriello MG, Husain A, Sovak MA, Yi J and Nycum LR: OCEANS: a randomized, double-blind, placebo-controlled phase III trial of chemotherapy with or without bevacizumab in patients with platinum-sensitive recurrent epithelial ovarian, primary peritoneal, or fallopian tube cancer. J Clin Oncol 30(17): 20392045, 2012.

7 Pujade-Lauraine E, Hilpert F, Weber B, Reuss A, Poveda A, Kristensen G, Sorio R, Vergote I, Witteveen P, Bamias A, Pereira D, Wimberger P, Oaknin A, Mirza MR, Follana P, Bollag D and Ray-Coquard I: AURELIA: a randomized phase III trial evaluating bevacizumab (BEV) plus chemotherapy (CT) for platinum (PT)-resistant recurrent ovarian cancer (OC). J Clin Oncol 32(13): 1302-1308, 2014.

8 Monk BJ, Poveda A, Vergote I, Raspagliesi F, Fujiwara K, Bae DS, Oaknin A, Ray-Coquard I, Provencher DM, Karlan BY, Lhommé C, Richardson G, Rincón DG, Coleman RL, Herzog TJ, Marth C, Brize A, Fabbro M, Redondo A, Bamias A, Tassoudji M, Navale L, Warner DJ and Oza AM: Antiangiopoietin therapy with trebananib for recurrent ovarian cancer (TRINOVA-1): a randomised, multicentre, double-blind, placebo-controlled phase 3 trial. Lancet Oncol 15(8): 799-808, 2014.

9 Monk BJ, Poveda A, Vergote I, Raspagliesi F and Fujiwara K: Impact of trebananib plus weekly paclitaxel on overall survival in patients with recurrent ovarian cancer and ascites: results from the phase III TRINOVA-1 study. J Clin Oncol 33: abtract 5503, 2015.

10 Tew WP, Colombo N, Ray-Coquard I, Del Campo JM, Oza A, Pereira D, Mammoliti S, Matei D, Scambia G, Tonkin K, Shun Z, Sternas L and Spriggs DR: Intravenous aflibercept in patients with platinum-resistant, advanced ovarian cancer: results of a randomized, double-blind, phase 2, parallel-arm study. Cancer 120(3): 335-343, 2014.

11 Sonpavde G, Hutson TE and Sternberg CN: Pazopanib, a potent orally administered small-molecule multitargeted tyrosine kinase inhibitor for renal cell carcinoma. Expert Opin Investig Drugs 17(2): 253-261, 2008.

12 Pignata S, Lorusso D, Scambia G, Sambataro D, Tamberi S, Cinieri S, Mosconi AM, Orditura M, Brandes AA, Arcangeli V, Panici PB, Pisano C, Cecere SC, Di Napoli M, Raspagliesi F, Maltese G, Salutari V, Ricci C, Daniele G, Piccirillo MC, Di Maio M, Gallo C, Perrone F and MITO 11 investigators: Pazopanib plus weekly paclitaxel versus weekly paclitaxel alone for platinum-resistant or platinum-refractory advanced ovarian cancer (MITO 11): a randomised, open-label, phase 2 trial. Lancet Oncol 16(5): 561-568, 2015.

13 Friedlander M, Hancock KC, Rischin D, Messing MJ, Stringer CA, Matthys GM, Ma B, Hodge JP and Lager JJ: A Phase II, open-label study evaluating pazopanib in patients with recurrent ovarian cancer. Gynecol Oncol 119(1): 32-37, 2010.
$14 \mathrm{du}$ Bois A, Floquet A, Kim JW, Rau J, del Campo JM, Friedlander M, Pignata S, Fujiwara K, Vergote I, Colombo N, Mirza MR, Monk BJ, Kimmig R, Ray-Coquard I, Zang R, DiazPadilla I, Baumann KH, Mouret-Reynier MA, Kim JH, Kurzeder C, Lesoin A, Vasey P, Marth C, Canzler U, Scambia G, Shimada M, Calvert P, Pujade-Lauraine E, Kim BG, Herzog TJ, Mitrica I, Schade-Brittinger C, Wang Q, Crescenzo R and Harter P: Incorporation of pazopanib in maintenance therapy of ovarian cancer. J Clin Oncol 32(30): 3374-3382, 2014.

15 du Bois A, Kristensen G, Ray-Coquard I, Reuss A, Pignata S, Colombo N, Denison U, Vergote I, Del Campo JM, Ottevanger P, Heubner M, Minarik T, Sevin E, de Gregorio N, Bidziński M, Pfisterer J, Malander S, Hilpert F, Mirza MR, Scambia G, Meier W, Nicoletto MO, Bjørge L, Lortholary A, Sailer MO, Merger M, Harter P and AGO Study Group led Gynecologic Cancer Intergroup/European Network of Gynaecologic Oncology Trials Groups Intergroup Consortium: Standard first-line chemotherapy with or without nintedanib for advanced ovarian cancer (AGOOVAR 12): a randomised, double-blind, placebo-controlled phase 3 trial. Lancet Oncol 17(1): 78-89, 2016.

16 Raja FA, Griffin CL, Qian W, Hirte H, Parmar MK, Swart AM and Ledermann JA: Initial toxicity assessment of ICON6: a randomised trial of cediranib plus chemotherapy in platinum-sensitive relapsed ovarian cancer. Br J Cancer 105(7): 884-889, 2011.

17 Ledermann JA, Perren T, Raja FA, Embleton A, Rustin GJS, Jayson G, Kaye SB, Swart A, Vaughan M and Hirte H: Randomised double-blind phase III trial of cediranib (AZD 2171 ) in relapsed platinum sensitive ovarian cancer: results of the ICON6 trial. Eur J Cancer 49:abstrLBA10, 2013.

18 Liu JF, Barry WT, Birrer M, Lee JM, Buckanovich RJ, Fleming GF, Rimel B, Buss MK, Nattam S, Hurteau J, Luo W, Quy P, Whalen C, Obermayer L, Lee H, Winer EP, Kohn EC, Ivy SP and Matulonis U:. Combination cediranib and olaparib versus olaparib alone for women with recurrent platinum-sensitive ovarian cancer: a randomised phase 2 study. Lancet Oncol 15(11):1207-1214, 2014.

19 Mendel DB, Laird AD, Xin X, Louie SG, Christensen JG, Li G, Schreck RE, Abrams TJ, Ngai TJ, Lee LB, Murray LJ, Carver J, Chan E, Moss KG, Haznedar JO, Sukbuntherng J, Blake RA, Sun L, Tang C, Miller T, Shirazian S, McMahon G and Cherrington JM: In vivo antitumor activity of SU11248, a novel tyrosine kinase inhibitor targeting vascular endothelial growth factor and platelet-derived growth factor receptors: determination of a pharmacokinetic/pharmacodynamic relationship. Clin Cancer Res 9(1): 327-337, 2003.

20 Wilhelm S, Carter C, Lynch M, Lowinger T, Dumas J, Smith RA, Schwartz B, Simantov R and Kelley S: Discovery and development of sorafenib: a multikinase inhibitor for treating cancer. Nat Rev Drug Discov 5(10): 835-844, 2006.

21 Chan DW, Hui WW, Cai PC, Liu MX, Yung MM, Mak CS, Leung TH, Chan KK and Ngan HY: Targeting GRB7/ERK/ FOXM1 signaling pathway impairs aggressiveness of ovarian cancer cells. PLoS One 7(12): e52578, 2012.

22 Beach JA, Aspuria PJ, Cheon DJ, Lawrenson K, Agadjanian H, Walsh CS, Karlan BY and Orsulic S: Sphingosine kinase 1 is required for TGF- $\beta$ mediated fibroblastto-myofibroblast differentiation in ovarian cancer. Oncotarget 7(4): 4167-4182, 2016.

23 Konecny GE: Cyclin-dependent kinase pathways as targets for women's cancer treatment. Curr Opin Obstet Gynecol 28(1): 4248,2016 . 
24 Liu M, Qi Z, Liu B, Ren Y, Li H, Yang G and Zhang Q: RY-2f, an isoflavone analog, overcomes cisplatin resistance to inhibit ovarian tumorigenesis via targeting the $\mathrm{PI} 3 \mathrm{~K} / \mathrm{AKT} / \mathrm{mTOR}$ signaling pathway. Oncotarget 6(28): 25281-25294, 2015.

25 Gritsina G, Xiao F, O’Brien SW, Gabbasov R, Maglaty MA, Xu RH, Thapa RJ, Zhou Y, Nicolas E, Litwin S, Balachandran S, Sigal LJ, Huszar D and Connolly DC: Targeted Blockade of JAK/STAT3 Signaling Inhibits Ovarian Carcinoma Growth. Mol Cancer Ther 14(4): 1035-1047, 2015.

26 Cheaib B, Auguste A and Leary A: The PI3K/Akt/mTOR pathway in ovarian cancer: therapeutic opportunities and challenges. Chin J Cancer 34(1): 4-16, 2015.

27 Ueno NT, Bartholomeusz C, Herrmann JL, Estrov Z, Shao R, Andreeff M, Price J, Paul RW, Anklesaria P, Yu D and Hung MC: E1A-mediated paclitaxel sensitization in HER-2/neuoverexpressing ovarian cancer SKOV3.ip1 through apoptosis involving the caspase-3 pathway. Clin Cancer Res 6(1): 250-259, 2000.

28 Ma X, Wang S, Zhou J, Xing H, Xu G, Wang B, Chen G, Lu YP and Ma D: Induction of apoptosis in human ovarian epithelial cancer cells by antisurvivin oligonucleotides. Oncol Rep 14(1): 275-279, 2005 .
29 Bräutigam K, Bauerschlag DO, Weigel MT, Biernath-Wüpping J, Bauknecht T, Arnold N, Maass N and Meinhold-Heerlein I: Combination of enzastaurin and pemetrexed inhibits cell growth and induces apoptosis of chemoresistant ovarian cancer cells regulating extracellular signal-regulated kinase $1 / 2$ phosphorylation. Transl Oncol 2(3): 164-173, 2009.

30 Yasmeen A, Beauchamp MC, Piura E, Segal E, Pollak M, Gotlieb WH. Induction of apoptosis by metformin in epithelial ovarian cancer: involvement of the Bcl-2 family proteins. Gynecol Oncol 121(3): 492-498, 2011.

31 Wang J, Hansen K, Edwards R, Van Houten B and Qian W: Mitochondrial division inhibitor 1 (mdivi-1) enhances death receptor-mediated apoptosis in human ovarian cancer cells. Biochem Biophys Res Commun 456(1): 7-12, 2015.

Received March 23, 2017

Revised April 13, 2017

Accepted April 20, 2017 